\title{
Efeitos terapêuticos da produção artística para a reabilitação psicossocial de pessoas com transtornos mentais: uma revisão sistemática da literatura
}

\author{
Therapeutic effects of art making in the psychosocial \\ rehabilitation of people with mental disorders: \\ systematic literature review
}

\author{
Pedro Rocha Correia¹, Mônica de Oliveira Nunes de Torrenté
}

\begin{abstract}
Resumo
Atividades criativas vêm sendo utilizadas como recurso terapêutico para portadores de transtornos psíquicos no Brasil e no mundo. Há a necessidade de se consolidar a base teórica sobre o tema, levando-se em consideração dados qualitativos e o modelo conceitual do recovery. Neste trabalho, foi realizada uma revisão sistemática sobre os efeitos da produção de arte como recurso terapêutico no campo da saúde mental. Foram pesquisadas as bases de dados PubMed, LILACS e SciELO, com a seleção de estudos de metodologia qualitativa sobre o tema, publicados em inglês ou português, entre 2000 e 2013. Vinte e oito artigos foram revisados por meio de análise de conteúdo. Foram demonstrados benefícios para aspectos importantes do processo de reabilitação dos sujeitos, como o alívio de sentimentos negativos, o empoderamento e a reinserção social, fundamentais ao modelo do recovery. Conclui-se que a arte possui um potencial terapêutico significativo para a reabilitação psicossocial de pessoas com transtornos psíquicos, podendo funcionar como um recurso valioso no cenário atual de desafios para o cuidado na saúde mental. Palavras-chave: terapia pela arte; saúde mental; transtornos mentais; reabilitação.
\end{abstract}

\begin{abstract}
Creative activities have been used as therapeutic resource for people with mental disorders in Brazil and worldwide. There is a need to consolidate the body of evidence on the subject by incorporating qualitative data and the conceptual model of recovery. This paper presents a systematic review on the effects of art making as therapeutic resource in mental health. The databases PubMED, LILACS and SciELO were searched and studies on the subject that had qualitative approach and were published in English or Portuguese between 2000 and 2013 were selected. Benefits were evident in key aspects of the subjects' rehabilitation process, such as the relief of negative feelings, empowerment and social reintegration, which are fundamental principles of recovery. It was found that art has a significant therapeutic potential in the psychosocial rehabilitation of people with mental disorders and may represent a valuable resource in the current scenario of challenges to mental health care.
\end{abstract}

Keywords: art therapy; mental health; mental disorders; rehabilitation.

\footnotetext{
${ }^{1}$ Faculdade de Medicina da Bahia, Universidade Federal da Bahia (UFBA) - Salvador (BA), Brasil.

${ }^{2}$ Instituto de Saúde Coletiva, Universidade Federal da Bahia (UFBA) - Salvador (BA), Brasil.

Trabalho realizado na Universidade Federal da Bahia (UFBA) - Salvador (BA), Brasil.

Endereço para correspondência: Pedro Rocha Correia - Faculdade de Medicina da Bahia, Universidade Federal da Bahia (UFBA), Av. Reitor Miguel Calmon, s/n Vale do Canela - CEP: 40110-100 - Salvador, (BA), Brasil - Email: pedrorocha.cs@gmail.com

Fonte de financiamento: nenhuma.
}

Conflito de interesses: nada a declarar. 


\section{INTRODUÇÃO}

Os movimentos de Reforma Psiquiátrica propiciaram, na segunda metade do século XX, a ascensão de um novo paradigma na assistência a portadores de transtornos mentais ${ }^{1}$. O conceito de recovery, proposto a partir da década de $1970^{2}$, insere-se nesse contexto de reconstrução das práticas assistenciais, ao lançar um modelo inovador de reabilitação para pessoas com transtornos psíquicos.

Criado a partir de narrativas de usuários sobre suas experiências como portadores de doenças mentais ${ }^{3}$, o recovery se propõe a substituir a reabilitação centrada em conceitos biomédicos, incorporando dimensões subjetivas ao cuidado em saúde mental ${ }^{4}$. O modelo descreve a reabilitação do sujeito como um processo profundamente pessoal de autodeterminação, fortalecimento e descoberta de um novo senso de identidade e propósito, contemplando a superação do rótulo de louco ${ }^{3-6}$. O recovery não preconiza, portanto, cura ou remissão de sintomas ${ }^{2}$, mas, ao invés de disso, centra-se na recuperação da esperança por parte do usuário ${ }^{2,3}$ e no viver bem, apesar do transtorno psíquico ${ }^{7}$.

$\mathrm{O}$ uso da arte como recurso terapêutico se insere nesse contexto de busca por soluções que atendam às demandas dos novos modelos de reinserção social. Práticas artísticas vêm sendo empregadas em instituições de diversos países ${ }^{8-11}$ e, hoje, ocupam papel de destaque entre as atividades terapêuticas oferecidas nos Centros de Atenção Psicossocial (CAPS) ${ }^{12}$. Embora alguns estudos quantitativos com amostras significativas não tenham demonstrado benefícios da arteterapia para portadores de transtornos mentais ${ }^{13,14}$, publicações com metodologia qualitativa têm mostrado resultados diferentes, com repercussões positivas em diversas frentes da reabilitação ${ }^{15}$. Apesar de a pesquisa qualitativa ser mais condizente com os princípios do recovery, essa abordagem metodológica foi pouco explorada em revisões anteriores sobre o tema ${ }^{15}$. Assim, os efeitos terapêuticos dessas práticas não estão completamente elucidados ${ }^{13,14}$, o que tem sido apontado como um entrave ao financiamento e à incorporação dessas práticas aos serviços de saúde mental ${ }^{15-17}$. O presente estudo localizou apenas uma revisão publicada sobre o tema no Brasil'.

O presente estudo tem por objetivo identificar e discutir efeitos benéficos da arte para os processos de reabilitação psicossocial ou recovery de portadores de transtornos psíquicos, relacionando os efeitos descritos a eventuais explicações propostas pelos estudos. A revisão se justifica pela necessidade de se consolidar o corpo de evidências nesse campo ${ }^{15-17}$ por meio de metodologia qualitativa e à luz de modelos de reabilitação inovadores ${ }^{18,19}$.

\section{METODOLOGIA}

Foi realizada uma revisão sistemática da literatura sobre os efeitos terapêuticos da produção de arte para portadores de transtornos mentais. Foram incluídos na seleção artigos originais em português e em inglês, publicados entre os anos de 2000 e 2013 e que avaliaram os efeitos do uso da arte como recurso terapêutico para pessoas com transtornos mentais por meio de metodologia qualitativa. Não foram incluídos: (i) estudos que avaliaram os efeitos dessas intervenções para pacientes com dependência de substâncias psicoativas e demência; (ii) estudos com metodologia mista (quantitativa e qualitativa); (iii) artigos que não puderam ser recuperados na íntegra. Para o levantamento bibliográfico, foi realizada uma busca nos bancos de dados Medical Literature Analysis and Retrieval System Online (MEDLINE/PubMed), Literatura Latino-Americana e do Caribe em Ciências da Saúde (LILACS) e Scientific Electronic Library Online (SciELO). Os descritores foram divididos em duas categorias: art making, art therapy e creative therapy/creative therapies (categoria 1); mental disorders, mental health e mental illness (categoria 2). Desses termos, art therapy, mental disorders e mental health são termos indexados no Medical Subject Headings (MeSH) e nos Descritores em Ciências da Saúde da Biblioteca Virtual de Saúde (DeCS/BIREME). Para elaborar a sintaxe da busca, cada termo da categoria 1 foi combinado com um termo da categoria 2 até que todas as combinações tivessem sido realizadas. O termo art therapy foi pesquisado entre aspas, pois, nas tentativas de busca sem esse recurso, a maioria dos artigos encontrados abordavam temas que não interessavam a esta revisão.

$\mathrm{Na}$ base de dados PubMed, uma das estratégias teve de ser modificada. Nas buscas com o termo "art therapy", 1.066 artigos foram localizados, em muitos dos quais o tema era a terapia antirretroviral, devido à semelhança de art therapy com a sigla para antiretroviral (art) therapy (terapia antirretroviral, utilizada no tratamento da infecção pelo vírus HIV). Assim, acrescentou-se o termo "NOT HIV" à busca. Ainda, nas buscas no PubMed, foram utilizados os seguintes filtros: idiomas inglês e português, e artigos publicados entre $1^{\circ}$ de janeiro de $2000 \mathrm{e}$ 31 de dezembro de 2013.

As buscas foram realizadas entre junho e julho de 2014 . Para buscar artigos indisponíveis nas bases de dados listadas anteriormente, foram pesquisadas a Biblioteca Virtual de Saúde (BVS), por meio do portal da Biblioteca Regional de Medicina (BIREME), e, nos casos de teses e dissertações, o banco de teses da Coordenação de Aperfeiçoamento de Pessoal de Nível Superior (CAPES).

Os resultados dos artigos selecionados foram categorizados a partir do método da análise de conteúdo ${ }^{20}$. Para o processo de categorização, os 28 artigos foram lidos novamente, destacando-se trechos que indicavam benefícios da arte para as trajetórias de pessoas com transtornos mentais. Dos trechos escolhidos, foram extraídos significados que sintetizassem a natureza dos efeitos relatados e que foram então agrupados de acordo com o tema ao qual se referiam. Desse modo, foram construídas 
categorias de análise, que formaram a base da análise realizada pela presente revisão.

\section{RESULTADOS}

Vinte e oito artigos foram revisados. O processo de seleção de artigos pode ser visualizado na Figura 1.

Entre os estudos incluídos na presente revisão (Tabela 1), mais da metade (15) consistiu em trabalhos exploratórios de natureza qualitativa, nove artigos eram relatos de experiência e os demais artigos (quatro) eram relatos ou séries de casos.

Os métodos de amostragem e critérios de elegibilidade para a participação nos estudos não foram descritos em todos os artigos. Houve extensa variabilidade no modo de caracterizar as populações estudadas. Apenas 10 dos artigos revisados especificaram os diagnósticos psiquiátricos dos participantes da pesquisa, que incluíram: depressão maior unipolar, transtorno bipolar, esquizofrenia, transtornos alimentares, transtorno obsessivo-compulsivo, transtorno de estresse pós-traumático e transtornos de personalidade. Outras características apresentadas pelos estudos foram sexo dos participantes (em 19 artigos), idade (em 11 artigos) e etnia (em cinco artigos).

As modalidades artísticas e o número de artigos em que elas foram relatadas como predominantes entre as atividades

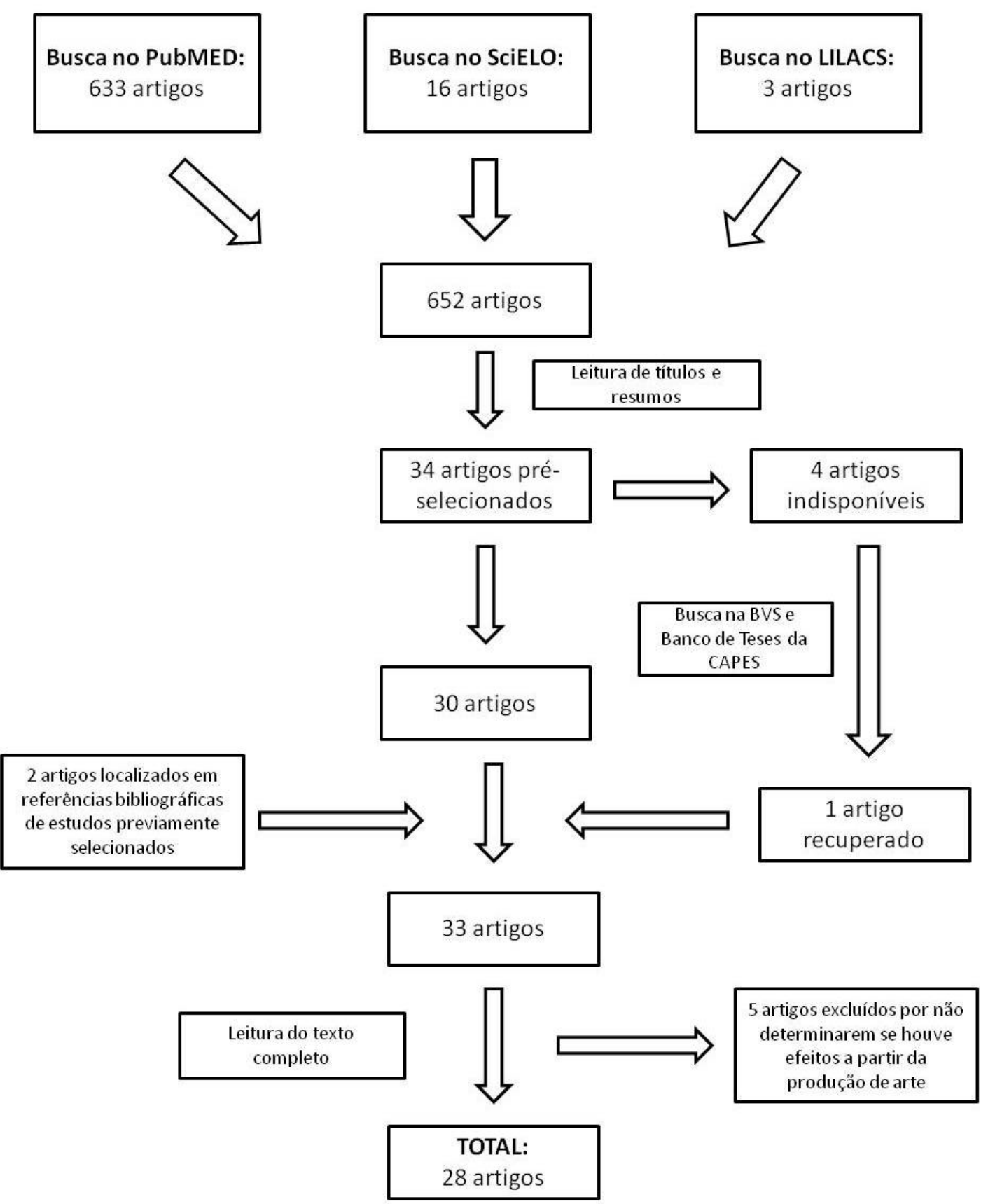

Figura 1. Processo de seleção dos artigos 
Tabela 1. Caracterização dos artigos selecionados quanto ao ano, autor, país de origem, modalidade artística investigada, modo de desenvolvimento da atividade, efeitos descritos e modo de avaliação dos efeitos

\begin{tabular}{|c|c|c|c|c|}
\hline Autor e país & $\begin{array}{l}\text { Modalidade } \\
\text { artística } \\
\text { predominante }\end{array}$ & $\begin{array}{c}\text { Atividade } \\
\text { desenvolvida } \\
\text { individualmente } \\
\text { ou em grupo? }\end{array}$ & Efeitos relatados & $\begin{array}{l}\text { Leva em conta } \\
\text { a perspectiva } \\
\text { dos usuários na } \\
\text { avaliação dos } \\
\text { efeitos? }\end{array}$ \\
\hline Avrahami $^{21} /$ Israel & Artes plásticas & Individualmente & $\begin{array}{l}\text { Expressividade, revisão da identidade, } \\
\text { empoderamento, reconquista da } \\
\text { esperança e concretização de planos }\end{array}$ & Sim \\
\hline Camargo et al. ${ }^{22}$ / Brasil & Artes plásticas & Em grupo & Expressividade e revisão da identidade & Sim \\
\hline Clark $^{23} /$ Austrália & Poesia & Não determinado & $\begin{array}{l}\text { Expressividade e minimização de } \\
\text { aspectos negativos da doença mental }\end{array}$ & Sim \\
\hline Coqueiro et al. ${ }^{24}$ / Brasil & Artes plásticas & Em grupo & $\begin{array}{l}\text { Revisão da identidade e minimização } \\
\text { de aspectos negativos da doença mental }\end{array}$ & Não especificado \\
\hline Giles et al. ${ }^{25}$ / Canadá & Não determinado & Em grupo & $\begin{array}{l}\text { Revisão da identidade, empoderamento, } \\
\text { sociabilidade, minimização de } \\
\text { aspectos negativos da doença mental e } \\
\text { sociabilidade }\end{array}$ & Sim \\
\hline Galvanese et al. ${ }^{1}$ / Brasil & Artes plásticas & Em grupo & $\begin{array}{l}\text { Revisão da identidade, ampliação } \\
\text { de competências pessoais, } \\
\text { empoderamento, reconquista da } \\
\text { esperança e sociabilidade }\end{array}$ & Não \\
\hline Glaister $^{26}$ / Estados Unidos & Artes plásticas & Individualmente & $\begin{array}{l}\text { Revisão da identidade e } \\
\text { empoderamento }\end{array}$ & Sim \\
\hline Goodsmith $^{27}$ / Estados Unidos & Fotografia & Em grupo & $\begin{array}{l}\text { Revisão da identidade, ampliação } \\
\text { de competências pessoais, } \\
\text { empoderamento, reconquista da } \\
\text { esperança, sociabilidade e minimização } \\
\text { de aspectos negativos da doença mental }\end{array}$ & Sim \\
\hline Howells e Zelnik ${ }^{28}$ / Estados Unidos & Artes plásticas & Em grupo & $\begin{array}{l}\text { Revisão da identidade, concretização de } \\
\text { planos, sociabilidade e minimização de } \\
\text { aspectos negativos da doença mental }\end{array}$ & Sim \\
\hline Karlsson e Malmqvist ${ }^{29}$ / Suécia & Não determinado & Individualmente & $\begin{array}{l}\text { Expressividade, revisão da identidade, } \\
\text { ampliação de competências pessoais, } \\
\text { empoderamento e minimização de } \\
\text { aspectos negativos da doença mental }\end{array}$ & Sim \\
\hline Lamont et al. ${ }^{30} /$ Austrália & Artes plásticas & Individualmente & $\begin{array}{l}\text { Expressividade e minimização de } \\
\text { aspectos negativos da doença mental }\end{array}$ & Sim \\
\hline Lloyd et al. ${ }^{4}$ / Austrália & Artes plásticas & Em grupo & $\begin{array}{l}\text { Expressividade, revisão da identidade, } \\
\text { ampliação de competências pessoais, } \\
\text { empoderamento, reconquista da } \\
\text { esperança, concretização de planos, } \\
\text { sociabilidade e minimização de } \\
\text { aspectos negativos da doença mental }\end{array}$ & Sim \\
\hline Makin e Gask ${ }^{31} /$ Reino Unido & Artes plásticas & Em grupo & $\begin{array}{l}\text { Expressividade, ampliação } \\
\text { de competências pessoais, } \\
\text { empoderamento, reconquista da } \\
\text { esperança, concretização de planos, } \\
\text { sociabilidade e minimização de } \\
\text { aspectos negativos da doença mental }\end{array}$ & Sim \\
\hline Margrove et al. ${ }^{32}$ / Reino Unido & Artes plásticas & Em grupo & $\begin{array}{l}\text { Expressividade, revisão da identidade, } \\
\text { ampliação de competências pessoais, } \\
\text { empoderamento e sociabilidade }\end{array}$ & Não \\
\hline Monaghan $^{33} /$ Austrália & Não determinado & Individualmente & $\begin{array}{l}\text { Expressividade, revisão da identidade, } \\
\text { empoderamento e concretização de } \\
\text { planos }\end{array}$ & Sim \\
\hline O’Donovan ${ }^{34} /$ Austrália & Teatro & Em grupo & $\begin{array}{l}\text { Ampliação de competências pessoais, } \\
\text { empoderamento, concretização de } \\
\text { planos e sociabilidade }\end{array}$ & Sim \\
\hline
\end{tabular}


Tabela 1. Continuação...

\begin{tabular}{|c|c|c|c|c|}
\hline Autor e país & $\begin{array}{l}\text { Modalidade } \\
\text { artística } \\
\text { predominante }\end{array}$ & $\begin{array}{c}\text { Atividade } \\
\text { desenvolvida } \\
\text { individualmente } \\
\text { ou em grupo? }\end{array}$ & Efeitos relatados & $\begin{array}{l}\text { Leva em conta } \\
\text { a perspectiva } \\
\text { dos usuários na } \\
\text { avaliação dos } \\
\text { efeitos? }\end{array}$ \\
\hline Perry et al. ${ }^{35}$ / Reino Unido & Não determinado & Em grupo & $\begin{array}{l}\text { Empoderamento, sociabilidade e } \\
\text { minimização de aspectos negativos da } \\
\text { doença mental }\end{array}$ & Sim \\
\hline $\begin{array}{l}\text { Rafieyan e Ries }{ }^{36} / \text { Estados } \\
\text { Unidos }\end{array}$ & Música & Individualmente & $\begin{array}{l}\text { Expressividade e minimização de } \\
\text { aspectos negativos da doença mental }\end{array}$ & Não determinado \\
\hline Ribeiro $^{37}$ / Brasil & Não determinado & Em grupo & $\begin{array}{l}\text { Empoderamento, reconquista da } \\
\text { esperança, } \\
\text { concretização de planos e sociabilidade }\end{array}$ & Não determinado \\
\hline Spandler et al. $.^{38} /$ Reino Unido & Não determinado & Em grupo & $\begin{array}{l}\text { Revisão da identidade, ampliação } \\
\text { de competências pessoais, } \\
\text { empoderamento, reconquista da } \\
\text { esperança, concretização de planos, } \\
\text { sociabilidade e minimização de } \\
\text { aspectos negativos da doença mental }\end{array}$ & Sim \\
\hline Stacey e Stickley ${ }^{39}$ / Reino Unido & Não determinado & Não especificado & $\begin{array}{l}\text { Expressividade, empoderamento e } \\
\text { sociabilidade }\end{array}$ & Sim \\
\hline Stickley e Hui ${ }^{40-42}$ / Reino Unido & Não determinado & Em grupo & $\begin{array}{l}\text { Expressividade, ampliação } \\
\text { de competências pessoais, } \\
\text { empoderamento, reconquista da } \\
\text { esperança, sociabilidade e minimização } \\
\text { de aspectos negativos da doença mental }\end{array}$ & Sim \\
\hline Stickley et al. ${ }^{43} /$ Reino Unido & Não determinado & Em grupo & $\begin{array}{l}\text { Expressividade, revisão da identidade, } \\
\text { reconquista da esperança, sociabilidade } \\
\text { e minimização de aspectos negativos da } \\
\text { doença mental }\end{array}$ & Sim \\
\hline Stickley e Hui ${ }^{40-42}$ / Reino Unido & Não determinado & Em grupo & $\begin{array}{l}\text { Revisão da identidade, ampliação } \\
\text { de competências pessoais, } \\
\text { empoderamento, reconquista da } \\
\text { esperança, concretização de planos, } \\
\text { sociabilidade e minimização de } \\
\text { aspectos negativos da doença mental }\end{array}$ & Sim \\
\hline Stickley e Hui ${ }^{40-42} /$ Reino Unido & Não determinado & Em grupo & $\begin{array}{l}\text { Expressividade, ampliação } \\
\text { de competências pessoais, } \\
\text { empoderamento, reconquista da } \\
\text { esperança, concretização de planos, } \\
\text { sociabilidade e minimização de } \\
\text { aspectos negativos da doença mental }\end{array}$ & Não \\
\hline Tavares $^{44}$ / Brasil & Não especificado & Em grupo & $\begin{array}{l}\text { Expressividade, revisão da identidade e } \\
\text { empoderamento }\end{array}$ & Não \\
\hline Teglbjaerg ${ }^{45}$ / Dinamarca & Artes plásticas & Em grupo & $\begin{array}{l}\text { Expressividade, revisão da identidade, } \\
\text { empoderamento, sociabilidade e } \\
\text { minimização de aspectos negativos da } \\
\text { doença mental }\end{array}$ & Sim \\
\hline Van Lith et al. ${ }^{46} /$ Austrália & Artes plásticas & Em grupo & $\begin{array}{l}\text { Expressividade, ampliação } \\
\text { de competências pessoais, } \\
\text { empoderamento, reconquista da } \\
\text { esperança, concretização de planos, } \\
\text { sociabilidade e minimização de } \\
\text { aspectos negativos da doença mental }\end{array}$ & Sim \\
\hline
\end{tabular}

realizadas podem ser vistos na Tabela 2. Nota-se um predomínio pelas artes plásticas nas investigações. A maioria dos artigos investigou atividades artísticas desenvolvidas na comunidade (21 artigos) e em grupo (22 artigos).
Nos estudos revisados, houve uma heterogeneidade de metodologias utilizadas para avaliar os resultados (Tabela 3). A mais frequente nos artigos foi o uso de entrevistas, utilizada em 16 estudos. Os artigos descreveram entrevistas do tipo aberta, 
semiestruturada e estruturada, com diferentes atores do processo. Foram ouvidos: portadores de transtornos mentais, sujeitos que conduziram as oficinas de produção artística (profissionais de saúde ou artistas profissionais) e profissionais de saúde que encaminharam portadores de transtornos mentais para programas de produção artística. A maioria dos artigos (21)

Tabela 2. Modalidades artísticas e suas frequências nos artigos

Modalidade artística

Artes plásticas

Música

Teatro

Poesia

Fotografia

Não determinada
Número de artigos em que foi predominante

12

1

1

1

1

12

Tabela 3. Metodologias utilizadas nos artigos revisados

\begin{tabular}{lc} 
Metodologia & $\begin{array}{c}\text { Frequência } \\
\text { nos artigos }\end{array}$ \\
Entrevistas formais & 16 \\
Com usuários & 11 \\
Com profissionais que conduziram as práticas & 2 \\
Com profissionais que conduziram as práticas e & 2 \\
com usuários & \\
Com profissionais que recomendaram as práticas & 1 \\
Análise do processo criativo & 4 \\
Narrativas pessoais & 3 \\
Etnografia & 1 \\
Outros & 1 \\
Não determinado & 3 \\
\hline
\end{tabular}

levou em consideração a perspectiva dos usuários na avaliação dos efeitos investigados. Nos estudos que utilizaram entrevistas, predominou-se a avaliação pontual de resultados, com entrevistas realizadas em uma única ocasião.

Todos os artigos selecionados descreveram efeitos terapêuticos da produção artística para a reabilitação psicossocial de portadores de transtornos mentais. Os efeitos benéficos destacados pelos artigos foram agrupados em oito categorias, construídas a partir de uma análise de conteúdo dos resultados encontrados: expressividade, revisão da identidade, ampliação de competências pessoais, empoderamento, reconquista da esperança, concretização de planos, sociabilidade e minimização de aspectos negativos da doença mental. As categorias e o número de artigos em que elas foram descritas podem ser vistos na Figura 2. Além disso, dois artigos apontaram efeitos negativos das práticas.

$\mathrm{Na}$ categoria empoderamento, foram agrupadas as repercussões associadas à promoção da autonomia do usuário e ao protagonismo deste na própria vida e em sua trajetória de recovery, dentre as quais: aumento da autoestima e do valor próprio ${ }^{4,25,31,32,35,40,44}$, confiança na própria capacidade de resolução de problemas ${ }^{26,31,32,40-42,44,45}$ e desenvolvimento de estratégias próprias para enfrentamento da doença $a^{4,32,38}$. Ademais, alguns usuários afirmaram que passaram a se sentir no controle da própria vida ${ }^{4,46}$ e viram a produção artística como algo necessário para lidar com as limitações impostas pela doença ${ }^{4}$. Entre as explicações propostas para os efeitos observados, estiveram a convivência com pessoas que enfrentavam as mesmas dificuldades ${ }^{35}$ e a percepção do usuário de que ele foi capaz de realizar algo que não conseguia fazer antes ${ }^{4,38,39}$.

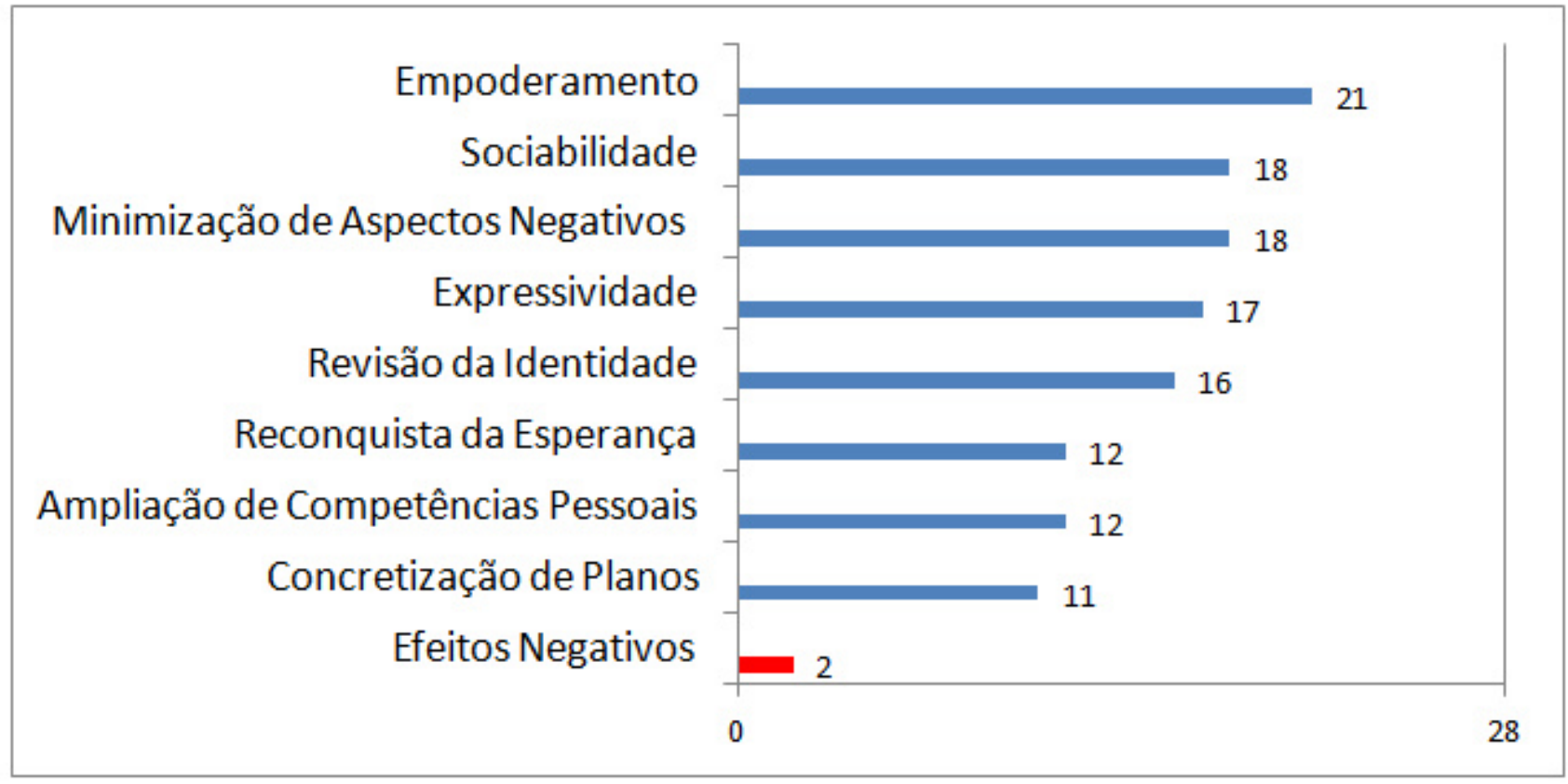

Figura 2. Categorias construídas e suas frequências nos artigos 
$\mathrm{Na}$ categoria sociabilidade, descreveram-se a interação e a formação de vínculo entre os usuários ${ }^{1,41,45}$, que permitiram o compartilhamento de ideias e de experiências ${ }^{1}$, e geraram oportunidades para conhecer pessoas com experiências similares $^{27,31}$. Destacaram-se o desenvolvimento de habilidades de comunicação, com diminuição da timidez e aumento da confiança ${ }^{4}$, e o surgimento de um senso de pertencimento social, uma vez que os usuários passaram a se sentir socialmente aceitos, incluídos e parte da comunidade ${ }^{40,42,45}$. A criação de uma cultura tolerante no grupo de atividades artísticas ${ }^{28}$ e a vivência em um ambiente seguro e não competitivo ${ }^{31}$ foram alguns dos mecanismos apontados para a obtenção dos efeitos.

A categoria minimização de aspectos negativos da doença mental contempla a vivência de dificuldades e de angústias de modo menos sofrido pelo portador de transtorno psíquico: a minimização de sentimentos e comportamentos negativos, como agressividade, agitação, ansiedade e pensamento paranoico ${ }^{24,29,30,36,43,45,46}$; o relaxamento a partir da atividade ${ }^{31,38,40-43}$; a diminuição do isolamento físico e psicológico ${ }^{27,28,35}$. Foram descritos ainda a amenização de comportamentos de automutilação ${ }^{38}$ e a diminuição do número de hospitalizações dos usuários ${ }^{4}$. Segundo os estudos, contribuíram para esses efeitos a intensa imersão nas atividades ${ }^{31,38,41}$, que permitiu a distração de dificuldades e de sentimentos negativos ${ }^{29,30}$, e o fato de que, durante as intervenções, deu-se mais ênfase às forças do que aos defeitos dos sujeitos ${ }^{29}$.

$\mathrm{Na}$ categoria expressividade, os efeitos mais destacados foram a expressão de memórias e de sentimentos negativos ou opressivos ${ }^{21,23,30,32,33}$ e a menor dificuldade para falar de si, usando as obras produzidas como facilitadoras ${ }^{22,30,32,40-45}$. Os benefícios conquistados facilitaram a comunicação entre paciente e equipe de saúde $e^{30,36,44}$, com melhora do vínculo entre usuários e profissionais, maior entendimento das necessidades do paciente e construção de um plano terapêutico mais efetivo ${ }^{30}$.

A autodescoberta ${ }^{4,24,27,32,42,45}$, o desenvolvimento de identidades renovadas, nas quais os usuários não eram definidos por sua doença mental ${ }^{38}$, e o processamento e ressignificação de experiências passadas ${ }^{25,29,32,45}$ foram alguns dos benefícios da categoria revisão da identidade. Alguns usuários passaram a se ver como artistas, o que contribuiu para a mudança do modo como se relacionavam com a comunidade e para a reconstrução de suas vidas ${ }^{28,38}$. Os efeitos foram atribuídos ao reconhecimento das conquistas dos usuários pelos pares, por profissionais e pela comunidade, com a exibição de obras ao público desempenhando um papel importante no processo $^{28,38}$.

$\mathrm{Na}$ categoria reconquista da esperança, destacaram-se a renovação da esperança no futuro ${ }^{31,38,40,43,46}$, a vontade de crescer e avançar na recuperação ${ }^{46}$, e o desenvolvimento de um senso de propósito na vida a partir da arte 4,38,42,46. $^{\text {. }}$
No entanto, dois artigos descreveram, de forma pontual, efeitos negativos da arte para a reabilitação dos sujeitos: uma percepção negativa da autoimagem ${ }^{35} \mathrm{e}$ as frustrações ligadas ao engajamento com produção artística, gerando raiva e isolamento ${ }^{43}$.

\section{DISCUSSÃO}

A presente revisão investigou os efeitos benéficos da produção de arte para a reabilitação psicossocial de pessoas com transtornos mentais, analisando os resultados à luz do conceito de recovery. Foram demonstrados benefícios para diversos aspectos do processo de reabilitação desses sujeitos, embora as categorias tenham variado quanto à frequência com que surgiram nos artigos e os benefícios tenham sido descritos com graus variados de intensidade.

A categoria de empoderamento foi a mais frequente nos estudos revisados e contemplou a aquisição da habilidade de entender e gerir a própria doença, a recuperação da possibilidade de tomar decisões e uma retomada do controle sobre a própria vida, o que se correlaciona com pontos-chave do conceito de recovery, como a promoção da autonomia e a construção de um autocuidado ${ }^{3}$. A interação com a comunidade foi um dos principais aspectos apontados como o responsável pela obtenção de efeitos nessa categoria, sugerindo que essa convivência é de fundamental importância para o processo de empoderamento do portador de transtorno mental.

Quanto à sociabilidade, observaram-se a reconstrução e a reconfiguração da rede social dos indivíduos, englobando a melhora das habilidades de relacionamento e a produção de vínculo, com consequente ampliação de suas redes sociais. Esses efeitos contribuem para a reinserção social do usuário, uma meta preconizada com ênfase pelo recovery, e são extremamente relevantes, uma vez que vulnerabilidade social é uma condição frequente entre portadores de transtornos mentais graves ${ }^{47}$. Em minimização de aspectos negativos da doença mental, foram descritos tanto remissão de sintomas como vivência da doença de modo menos sofrido pelo sujeito, o que atende a princípios fundamentais do recovery $y^{3}$.

Entre as explicações para a obtenção dos efeitos, destacou-se a formação de um "ambiente terapêutico" durante as atividades, que envolveu suporte mútuo entre os participantes ${ }^{27,40}$, atmosfera segura e tolerante ${ }^{28,35}$, e convivência em um grupo compreensivo ${ }^{46}$.

A diversidade dos benefícios descritos e a magnitude de alguns dos efeitos encontrados corroboram o valor que práticas de cuidado não médicas podem desempenhar no cuidado em saúde mental. No entanto, a descrição de efeitos negativos a partir das práticas impõe a necessidade de se investigar eventuais riscos dessas atividades. A durabilidade dos efeitos obtidos é outro aspecto importante a ser discutido, mas, devido ao predomínio de entrevistas pontuais nos artigos, não foi possível chegar a conclusões sobre esse tópico. 
Quanto às limitações da presente revisão, não foi realizada uma avaliação da qualidade dos estudos antes de selecioná-los para o trabalho. Além disso, nos artigos revisados, a heterogeneidade marcante e a superficialidade na descrição dos participantes dificultaram a comparação entre os diferentes estudos. Isso impediu constatações sobre características do coletivo de sujeitos de pesquisa que poderiam ser relevantes, como o seu diagnóstico psiquiátrico, idade, sexo e uso prévio ou concomitante de outras terapias, mas, especialmente, aspectos relacionados à sua biografia, posição social, nível educacional e cultura, todos fundamentais na contextualização dos resultados.

É relevante a participação de portadores de transtornos mentais na discussão e na construção dos estudos ${ }^{28,29,39,42}$, o que indica a inclusão da perspectiva do usuário, cuja voz tem sido historicamente negligenciada no processo de produção de conhecimento em Psiquiatria. A vivência singular de cada indivíduo com o engajamento em práticas artísticas, evidente nos artigos revisados, corrobora a importância do conceito de recovery na orientação da assistência em saúde mental e a necessidade de se pensar o cuidado nesse campo de forma singularizada, levando em consideração a experiência dos atores envolvidos no processo.

\section{CONCLUSÕES}

Foram identificados diversos benefícios para a reabilitação psicossocial de portadores de transtornos mentais a partir da produção de arte. Embora os usuários tenham descrito, pontualmente, dificuldades e aspectos negativos das atividades, estas tiveram amplo respaldo dos atores envolvidos no processo, o que permite reconhecer um enorme potencial a ser explorado a partir da arte como recurso terapêutico na saúde mental.

Alguns aspectos relativos ao uso da arte como recurso terapêutico para portadores de transtornos mentais precisam ser mais estudados, como os mecanismos pelos quais a arte promove seus efeitos, a durabilidade dos benefícios proporcionados e os possíveis riscos oferecidos pelo engajamento dos sujeitos com essas práticas.

\section{REFERÊNCIAS}

1. Galvanese ATC, Nascimento AF, D’Oliveira AFPL. Arte, cultura e cuidado nos centros de atenção psicossocial. Rev Saude Publica. 2013 abr;47(2):3607. PMid:24037364. http://dx.doi.org/10.1590/S0034-8910.2013047003487.

2. Baccari IOP, Campos RTO, Stefanello S. Revisão sistemática do conceito de recovery. Cien Saude Colet. 2015 jan;20(1):125-36. http://dx.doi. org/10.1590/1413-81232014201.04662013.

3. Duarte T. Recovery da doença mental: uma visão para os sistemas e serviços de saúde mental. Aná. Psicologica (Valencia). 2007 jan;25(1):127-33.

4. Lloyd C, Wong SR, Petchkovsky L. Art and recovery in mental health: a qualitative investigation. Br J Occup Ther. 2007 maio;70(5):207-14. http:// dx.doi.org/10.1177/030802260707000505.

5. Jaeger M, Hoff P. Recovery: conceptual and ethical aspects. Curr Opin Psychiatry. 2012 nov;25(6):497-502. PMid:22992552. http://dx.doi. org/10.1097/YCO.0b013e328359052f.

6. Coscrato G, Bueno SMV. A luz da arte nos Centros de Atenção Psicossocial: interface com o cuidado. Cad Bras Saude Mental. 2009 out/dez;1(2):142-50

7. Anthony WA. Recovery from mental illness: the guiding vision of the mental health service system in the 1990s. Psychosoc Rehabil J. 1993;16(4):521-38. http://dx.doi.org/10.1037/h0095655.

8. Tjörnstrand C, Bejerholm U, Eklund M. Participation in day centres for people with psychiatric disabilities: characteristics of occupations. Scand J Occup Ther. 2011 dez;18(4):243-53. PMid:21702742. http://dx.doi.org/ $10.3109 / 11038128.2011 .583938$

9. King R, Neilsen $P$, White E. Creative writing in recovery from severe mental illness. Int J Ment Health Nurs. 2013 out;22(5):444-52. PMid:23211053. http://dx.doi.org/10.1111/j.1447-0349.2012.00891.x.

10. Gajić GM. Group art therapy as adjunct therapy for the treatment of schizophrenic patients in day hospital. Vojnosanit Pregl. 2013;70(11):10659. PMid:24397206. http://dx.doi.org/10.2298/VSP1311065M

11. Bungay H, Clift S. Arts on prescription: a review of practice in the UK. Perspect Public Health. 2010 dez;130(6):277-81. PMid:21213564. http:// dx.doi.org/10.1177/1757913910384050.

12. Brasil. Ministério da Saúde. Saúde Mental no SUS: os Centros de Atenção Psicossocial. Brasília; 2004.

13. Crawford MJ, Killaspy H, Kalaitzaki E, Barrett B, Byford S, Patterson S, et al. The MATISSE study: a randomised trial of group art therapy for people with schizophrenia. BMC Psychiatry. 2010 ago;10(65):65. PMid:20799930. http://dx.doi.org/10.1186/1471-244X-10-65.

14. Ruddy R, Milnes D. Art therapy for schizophrenia or schizophrenia-like illnesses. Cochrane Database Syst Rev. 2005 out;(4):CD003728. PMid:16235338.

15. Van Lith T, Schofield MJ, Fenner P. Identifying the evidence-base for artbased practices and their potential benefit for mental health recovery: a critical review. Disabil Rehabil. 2013 ago;35(16):1309-23. PMid:23116320. http://dx.doi.org/10.3109/09638288.2012.732188.

16. Kilroy A, Garner C, Parkinson C, Kagan C, Senior P. Towards transformation: exploring the impact of culture, creativity and the arts on health and wellbeing. Manchester: Arts for Health, Manchester Metropolitan University; 2007.

17. Mental Health Foundation. Arts, creativity and mental health initiative: participatory Arts Self Evaluation Approach Project Report. Glasgow: Mental Health Foundation; 2007.

18. Campos GWS, Onocko-Campos RT, Del Barrio LD. Políticas e práticas em saúde mental: as evidências em questão. Cien Saude Colet. 2013 out;18(10):2797-805. http://dx.doi.org/10.1590/S1413-81232013001000002.

19. Anthony W, Rogers ES, Farkas M. Research on evidence-based practices: future directions in an era of recovery. Community Ment Health J. 2003 abr;39(2):101-14. PMid:12723845. http://dx.doi.org/10.1023/A:1022601619482.

20. Bauer MW, Gaskell G. Pesquisa qualitativa com texto, imagem e som: um manual prático. Petrópolis: Vozes; 2002. 
21. Avrahami D. Visual art therapy's unique contribution in the treatment of post-traumatic stress disorders. J Trauma Dissociation. 2005;6(4):5-38. PMid:16537321. http://dx.doi.org/10.1300/J229v06n04_02.

22. Camargo VP, Lena MS, Dias HZJ, Roso AR. Costurando saúde: possibilidades de integração por meio da confecção de bonecos(as) de pano em um CAPS infantil. Psicol. Argum. 2011 jan/mar;29(64):101-8.

23. Clark T. Poetry: recovery and beyond. Australas Psychiatry. 2009 fev; 17 (Supl 1):S167-9. PMid:19579135. http://dx.doi.org/10.1080/10398560902948407.

24. Coqueiro NF, Vieira FRR, Freitas MMC. Arteterapia como dispositivo terapêutico em saúde mental. Acta Paul Enferm. 2010;23(6):859-62. http:// dx.doi.org/10.1590/S0103-21002010000600022.

25. Giles MD, Nelson AL, Shizgal F, Stern E, Fourt A, Woods P, et al. A multimodal treatment program for childhood trauma recovery: Women Recovering from Abuse Program (WRAP). J Trauma Dissociation. 2007;8(4):7-24. PMid:18077282. http://dx.doi.org/10.1300/J229v08n04_02.

26. Glaister JA. Four years later: Clara revisited. Perspect Psychiatr Care. 2000 janmar;36(1):5-13. PMid:12035674. http://dx.doi.org/10.1111/j.1744-6163.2000. tb00684.x.

27. Goodsmith L. Beyond where it started: a look at the "Healing Images" experience. Torture. 2007;17(3):222-32. PMid:19289895.

28. Howells V, Zelnik T. Making art: a qualitative study of personal and group transformation in a community arts studio. Psychiatr Rehabil J. 2009;32(3):21522. PMid:19136354. http://dx.doi.org/10.2975/32.3.2009.215.222.

29. Karlsson LB, Malmqvist A. "Poetry in Yarn": making sense of life experiences in the shadow of Schizophrenia. Schizophr Bull. 2013 jul;39(4):732-6. PMid:23223341. http://dx.doi.org/10.1093/schbul/sbs144.

30. Lamont S, Brunero S, Sutton D. Art psychotherapy in a consumer diagnosed with borderline personality disorder: a case study. Int J Ment Health Nurs. 2009 jun;18(3):164-72. PMid:19490226. http://dx.doi.org/10.1111/j.14470349.2009.00594.x.

31. Makin S, Gask L. "Getting back to normal": the added value of an art-based programme in promoting "recovery" for common but chronic mental health problems. Chronic Illn. 2012 mar;8(1):64-75. PMid:21985790. http://dx.doi.org/10.1177/1742395311422613.

32. Margrove KL, Pope J, Mark GM. An exploration of artists' perspectives of participatory arts and health projects for people with mental health needs. Public Health. 2013 dez;127(12):1105-10. PMid:24275028. http://dx.doi. org/10.1016/j.puhe.2013.09.018.

33. Monaghan D. Creating an open mind. Australas Psychiatry. 2011 jul;19(Supl 1):S73-5. PMid:21878025. http://dx.doi.org/10.3109/10398562.2011.5830 52.

34. O'Donovan R. How the creative spirit saved me from a fate worse than hospitalization. Australas Psychiatry. 2011 jul;19(Supl 1):S65-8. PMid:21878023. http://dx.doi.org/10.3109/10398562.2011.583071.
35. Perry C, Thurston M, Osborn T. Time for Me: the arts as therapy in postnatal depression. Complement Ther Clin Pract. 2008 fev;14(1):38-45. PMid:18243941. http://dx.doi.org/10.1016/j.ctcp.2007.06.001.

36. Rafieyan R, Ries R. A description of the use of music therapy in consultationliaison psychiatry. Psychiatry. 2007 jan;4(1):47-52. PMid:20805929.

37. Ribeiro SFR. Grupo de expressão: uma prática em saúde mental. Rev. SPAGESP. 2007 jan/jun;8(1):25-35.

38. Spandler H, Secker J, Kent L, Hacking S, Shenton J. Catching life: the contribution of arts initiatives to recovery approaches in mental health. J Psychiatr Ment Health Nurs. 2007 dez;14(8):791-9. PMid:18039304. http:// dx.doi.org/10.1111/j.1365-2850.2007.01174.x.

39. Stacey G, Stickley T. The meaning of art to people who use mental health services. Perspect Public Health. 2010 mar;130(2):70-7. PMid:20455486. http://dx.doi.org/10.1177/1466424008094811.

40. Stickley T, Hui A. Social prescribing through arts on prescription in a UK city: referrers' perspectives (part 2). Public Health. 2012;126(7):580-6. PMid:22578297. http://dx.doi.org/10.1016/j.puhe.2012.04.001.

41. Stickley T, Hui A. Arts In-Reach: taking "bricks off shoulders" in adult mental health inpatient care. J Psychiatr Ment Health Nurs. 2012;19(5):4029. PMid:22070164. http://dx.doi.org/10.1111/j.1365-2850.2011.01811.x.

42. Stickley T, Hui A. Social prescribing through arts on prescription in a U.K. city: participants' perspectives (part 1). Public Health. 2012;126(7):574-9. PMid:22683358. http://dx.doi.org/10.1016/j.puhe.2012.04.002.

43. Stickley T, Hui A, Morgan J, Bertram G. Experiences and constructions of art: a narrative-discourse analysis. J Psychiatr Ment Health Nurs. 2007 dez;14(8):783-90. PMid:18039303. http://dx.doi.org/10.1111/j.13652850.2007.01173.x.

44. Tavares CMM. O papel da arte nos Centros de Atenção Psicossocial - CAPS. Rev Bras Enferm. 2003 jan/fev;56(1):35-9. PMid:14595971. http://dx.doi. org/10.1590/S0034-71672003000100007.

45. Teglbjaerg HS. Art therapy may reduce psychopathology in schizophrenia by strengthening the patients' sense of self: a qualitative extended case report. Psychopathology. 2011;44(5):314-8. PMid:21659793. http://dx.doi. org/10.1159/000325025

46. Van Lith T, Fenner P, Schofield M. The lived experience of art making as a companion to the mental health recovery process. Disabil Rehabil. 2011;33(8):652-60. PMid:20695818. http://dx.doi.org/10.3109/09638288 .2010 .505998 .

47. Muramoto MT, Mângia EF. A sustentabilidade da vida cotidiana: um estudo das redes sociais de usuários de serviço de saúde mental no município de Santo André (SP, Brasil). Cien Saude Colet. 2011 abr;16(4):2165-77. PMid:21584458. http://dx.doi.org/10.1590/S1413-81232011000400016.

Recebido em: Jul. 23, 2016 Aprovado em: Dez. 01, 2016 